\title{
Nutritional deficiencies in homeless persons with problematic drinking: a systematic review
}

Sharea ljaz ${ }^{1,2^{*}}$, Joni Jackson ${ }^{1,2}$, Helen Thorley ${ }^{1,2}$, Katie Porter $^{3}$, Clare Fleming ${ }^{4}$, Alison Richards ${ }^{1,2}$, Adrian Bonner $^{5}$ and Jelena Savović ${ }^{1,2}$

\begin{abstract}
Background: A significant proportion of homeless people drink alcohol excessively and this can lead to malnutrition and consequent medical problems. The aim of this review was to assess the evidence on the range of nutritional deficiencies in the homeless problem-drinking populations.

Methods: We conducted a comprehensive search of nine scientific literature databases and 13 grey literature sources. We included studies of any design that included homeless population with problem-drinking and reported measures of nutritional deficiencies in urine or blood. Study selection and data extraction was done by one reviewer and checked by another. Data on malnutrition profile were summarized narratively.

Results: We found nine studies reporting nutritional deficiencies in homeless populations with problem-drinking. The oldest study was from the 1950s and the most recent from 2013. The following nutrients were reported across studies: vitamins B1, B2, B6, B9, B12, C, A, and E; haemoglobin; and albumin. The most common deficiencies reported were of vitamin B1 (prevalence of deficiency was $0,2,6,45$, and $51 \%$ in five studies) and vitamin C (29, 84, and $95 \%$ in three studies). None of the studies were assessed to be at a low risk of bias.

Conclusions: The limited, low quality and relatively old evidence suggests that homeless people who drink heavily may be deficient in vitamin C, thiamine, and other nutrients. New, well conducted studies are needed in order to optimally inform public health interventions aimed at improving deficiencies in this population.
\end{abstract}

Trial Registration: PROSPERO CRD42015024247

Keywords: Homeless, Alcohol, Systematic review, Problem drinking, Malnutrition

\section{Background}

Nearly two thirds of homeless people in the UK drink more alcohol than is recommended [1]. The impact of homelessness on an individual, combined with the effects of excessive drinking can become a double blow on nutritional health and lead to deficiencies [2] (Fig. 1). These deficiencies contribute to neurological and other organ damage and can lead to long-lasting medical conditions [3].

\footnotetext{
*Correspondence: s.ijaz@bristol.ac.uk

${ }^{1}$ The National Institute for Health Research Collaboration for Leadership in Applied Health Research and Care West (NIHR CLAHRC West), University Hospitals Bristol NHS Foundation Trust, 9th Floor Whitefriars, Lewins Mead, Bristol BS1 2NT, UK

${ }^{2}$ School of Social and Community Medicine, University of Bristol, Bristol, UK Full list of author information is available at the end of the article
}

Historically vitamin B1 (thiamine) deficiency has been associated with homeless and problem-drinking populations and the observed neurological damage in these groups is attributed to its deficiency. Parenteral thiamine is therefore recommended for patients with suspected chronic alcohol misuse who are admitted to accident and emergency (A\&E) departments to prevent Wernicke's encephalopathy [4]. However, to our knowledge no comprehensive collation of evidence on the prevalence or severity of vitamin deficiencies in this population has been done recently. Knowing the range of the most common nutritional deficiencies can help develop an intervention to counter these deficiencies which can prevent illness in this high-risk group. In the long term, this could save costs for the health system, 


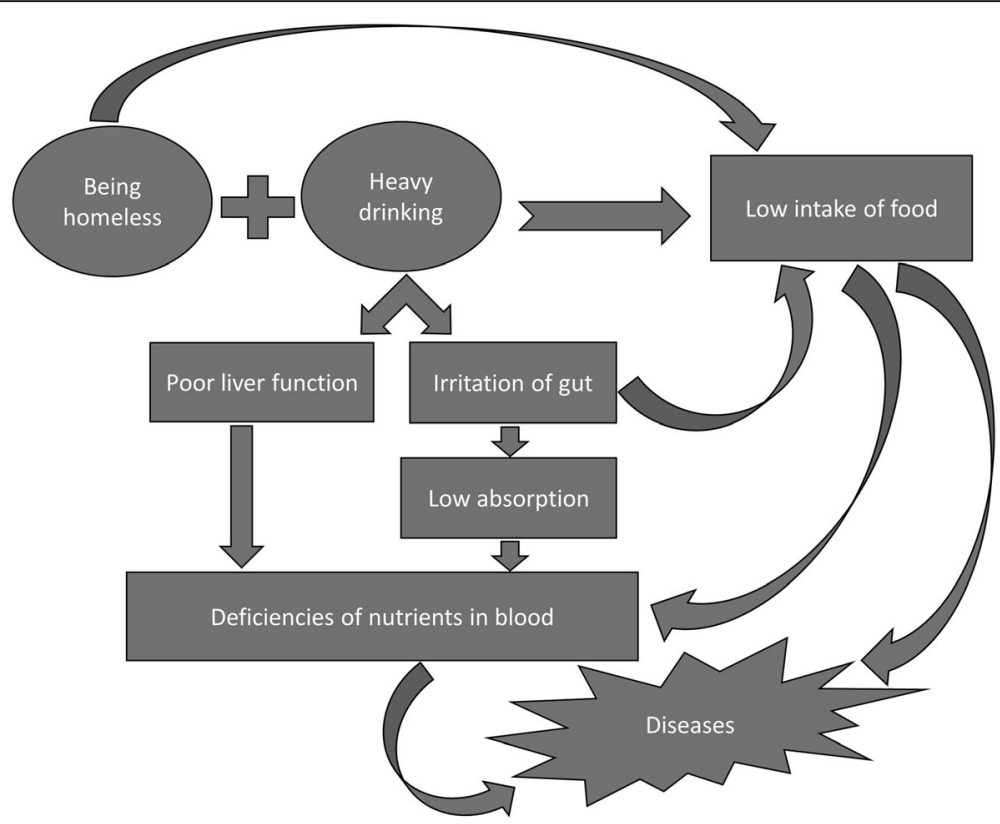

Fig. 1 Nutritional deficiency mechanisms in Homeless problem-drinkers

especially in A\&E attendances [5], as well as improve the health and quality of life of the homeless and the vulnerably housed people who consume alcohol excessively. This systematic review of literature was undertaken alongside a complementary systematic review of effectiveness of interventions for improving or preventing malnutrition in this population [6]. The aim of this review is to assess the evidence on prevalence and range of nutritional deficiencies in the homeless problemdrinking population. The findings of both reviews will inform the development of an intervention for nutritional deficiencies (e.g. supplements or fortified food products).

\section{Methods}

\section{Literature searches}

A single, comprehensive search strategy was developed and applied to multiple databases to find all relevant nutritional literature on homeless problem drinkers. This search strategy was used to identify studies, both for the current review of prevalence of malnutrition and the complementary review of intervention studies. The search was published in the protocol [6] and is up to date until 16th November 2016.

\section{Study inclusion and exclusion criteria}

We included studies of any design reporting nutrient levels in the homeless problem-drinking population. We included studies that fit the legal definition of homelessness used in the UK, which includes the following: sleeping rough (outside); residing in temporary accommodation such as hostels, bed and breakfasts, or night shelters; staying on a temporary basis with family or friends ('sofa surfers'); currently housed people who are at risk of being evicted; and currently housed people who cannot stay either: because they cannot afford to stay, or the home is in a very poor condition, or they are subject to violence, abuse or threats in the home [7]. Studies with participants of any age or gender were included as long as the majority of the population contained both problemdrinkers and homeless.

To be eligible studies had to report urinary or blood levels of any of the following nutrients: Vitamin A (Retinol, retinal, beta carotene), B1 (thiamine); B2 (riboflavin); B3 (niacin or nicotinic acid); B5 (pantothenic acid); B6 (pyridoxine, pyridoxal, pyridoxamine); B7 (biotin); B9 (folic acid), B12 (cobalamins), C (ascorbic acid), D (cholecalciferol/D3, ergocalciferol/D2), E (tocopherols, tocotrienols), K (phylloquinone, menaquinones), haemoglobin, albumin, ferritin, or iron.

We excluded the following: position papers; editorials; commentaries; studies where the participants were either homed alcohol drinkers or homeless without drinking problems; institutionalised people; studies where entire communities are homeless (e.g. occupiers of slums or shanty towns, refugees or similar populations); people homeless due to mass displacement (related to war, famine or natural disaster); non-nutrient outcomes (e.g. weight or BMI, muscle or fat mass); and studies of dietary intake (fruit/vegetables/fortified food/vitamins) as these are subject to recall bias and overlook the deficiency related to poor 
absorption or liver function - the consequences of problem-drinking.

At the screening stage [6], two reviewers independently tagged studies that were potentially relevant to the current and the intervention review. These tagged records were screened again by a third reviewer for relevance. In addition, the third reviewer independently screened the remaining untagged studies to ensure relevant papers were not missed. References of included primary studies as well as identified systematic reviews were searched to identify any additional studies. Identified relevant studies were assessed in full text for inclusion by one reviewer and the decision checked by another, with any disagreements resolved by a third reviewer.

\section{Data extraction strategy}

Data extraction was carried out by one reviewer on a pre-specified pro-forma in Microsoft Excel and checked by another. Disagreements were resolved by discussion with a third reviewer. Data were extracted for the following: author, year of publication, report type, year(s) of data collection, location of study, study design, aim, population, sample size, proportion of problem-drinkers and homeless in sample, ethnicity, gender, age, type of homelessness (all/shelter/rough sleeper etc.), deficiency measures and definitions reported in the study, method used to measure deficiency level, deficiencies of vitamins as reported (mean/SD; proportion deficient), and any other deficiency measures/outcomes reported.

\section{Risk of bias assessment}

There is no single widely accepted risk of bias tool for cross sectional or survey type studies of prevalence. We therefore considered the previous work in this area [8-11] and adopted some of the key criteria described in these studies. All these assessed three key areas of potential bias in prevalence studies: a representative and appropriately recruited sample, a valid and reliable measurement of the condition under study, and whether the analysis was adjusted for important covariates. We considered the following factors to be important confounding factors: age, sex, duration of homelessness/alcohol problems, and assessed these three areas of bias in our included studies. The overall risk of bias for a study was considered 'low' when the following three conditions were met: (i) all criteria related to the representativeness of study sample were judged to be at low risk of bias; (ii) at least 2 criteria related to measurement of deficiencies were judged to be at low risk; and (iii) the analysis was adjusted for important confounders.

\section{Data synthesis and presentation}

Data were tabulated for each nutrient. The included studies were very heterogeneous with respect to their population and time period so it was not considered appropriate to conduct formal meta-analyses and produce quantitative summary estimates of deficiencies across studies. Instead, we carried out a narrative synthesis. We did however produce forest plots without summary estimates to aid the presentation of the results (See Additional files 1 and 2).

\section{Results}

The search identified 6017 references. Of these 190 were initially tagged as potentially relevant for the current review and 19 further records were identified through rescreening. A total of 209 references were identified for full paper assessment. Of these, 14 papers (referring to nine studies) were included and 195 papers were excluded (Fig. 2). The online supplement contains the list of all excluded studies (Additional file 1).

Six of the included studies (reported in 11 papers) were surveys [12-22], two were case reports [23, 24], and one was an intervention study [16].

Most studies were small. The median number of participants included in studies was 39 (IQR 8-71). The total number of participants included in this review is 621. Three studies $[17,22,24]$ included ethnic Black participants while others provided no information on ethnicity. In the four studies that included women, they comprised a small proportion of the sample (between 6 and $13 \%)$. Age of the participants was more or less the same across the studies, ranging from 19 to 76 years with an average of 46 years (IQR 43.25-48.75). Participants recruited were either from the streets (rough sleepers) $[17,18,21,23,24]$, or from shelters and clinics $[13,16,18,22]$. When reported, $[18,21]$, the duration of homelessness varied greatly, ranging between one month to 55 years among the participants. Table 1 displays key characteristics of included studies.

\section{Risk of bias in included studies}

None of the included studies were at low risk of bias (Fig. 3). The risk of bias related to the representativeness of the sample was high in all but one study. The vitamin levels in the blood were usually assessed using valid and reliable methods across study participants. However, for most studies the analysis did not adjust for important covariates (age, sex, duration of homelessness/alcohol problems).

\section{Data synthesis}

\section{Deficiencies reported}

The most commonly reported data were vitamin B1 levels (6 studies [13, 16, 17, 19, 21, 22] followed by B6, 


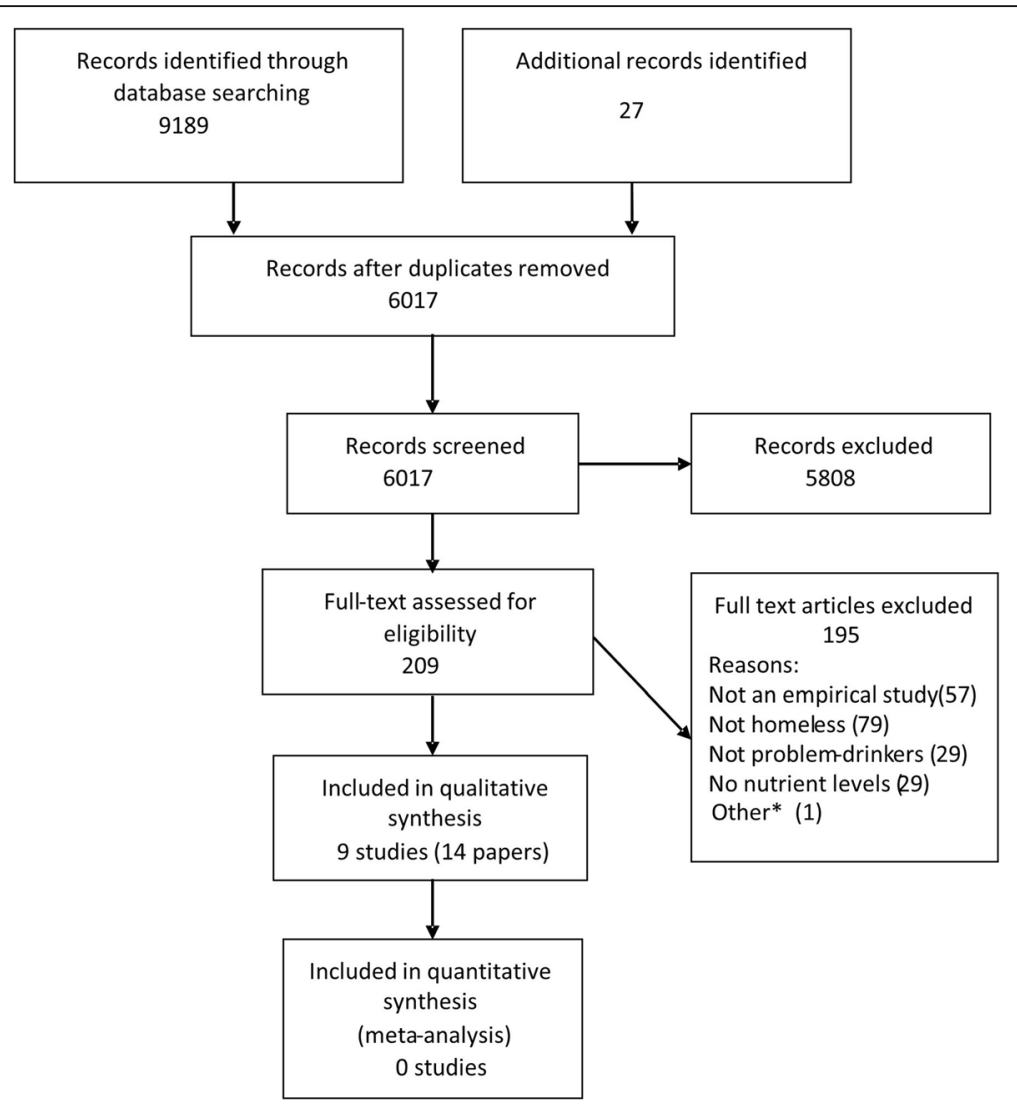

*TB treatment related malnutrition in a homeless person

Fig. 2 PRISMA flow diagram for review process

and B12 (5 studies [13, 16, 19, 21, 22]). Vitamin C, B9, and haemoglobin levels were reported in three studies, while vitamin A, B2, and E levels were reported in a single study each.. Vitamins B3, B5, B7, D, and K levels were not reported in any included studies.

The measurement methods were variable across studies. This probably reflects the advancement of laboratory techniques for assessment of nutrients over the past several decades. For example, the oldest study measured fasting excretion level in urine for the vitamins B1 and B2 and found that none of the participants were deficient in thiamine. In three later studies, vitamin B1 deficiency was measured using various measures in blood: the thiamine pyrophosphate (TTP) effect [13], the erythrocyte thiamine level [22], and the thiamine diphosphate (TDP) effect [16] and transketolase activity (TK activity) [13] and these studies used varied techniques and instruments for these measurements (Table 2).

The definitions or thresholds for deficiencies also varied. For example, for thiamine the following five definitions were used across studies: (i) excretion of less than $0.6 \mathrm{microgram} / \mathrm{hr}$ thiamine; (ii) thiamine pyrophosphate (TPP) effect for thiamine deficiency in range of 15 to 44 where $0-14$ is adequate; (iii) thiamine diphosphate (TDP)-effect for thiamine deficiency where reference range is $0-25 \%$; (iv) reference range from 59 to $213 \mathrm{mmol} / \mathrm{l}$; and (v) reference range from 50 to 106 microgram/litre. All the studies reported deficiencies as average (mean/median) levels for groups, with or without measures of spread (SD, IQR). Six studies also reported the proportion of people found deficient based on reference values for the general population.

\section{Evidence on deficiencies- Prevalence and severity}

The range of mean circulating vitamin levels observed across the included studies is presented in Table 3. No clear pattern of nutrient deficiency was observed across the studies for any of the vitamins. The mean levels of vitamins varied widely across the studies. For studies which reported data on the proportion of participants below deficiency thresholds, the most common deficiency was of vitamin C. All three studies that measured this factor found a significant proportion (29, 84, and 95\%) of participants were deficient. The other common deficiency was for vitamin B1, with two studies from the 1980 s reporting nearly half of the studied population to 


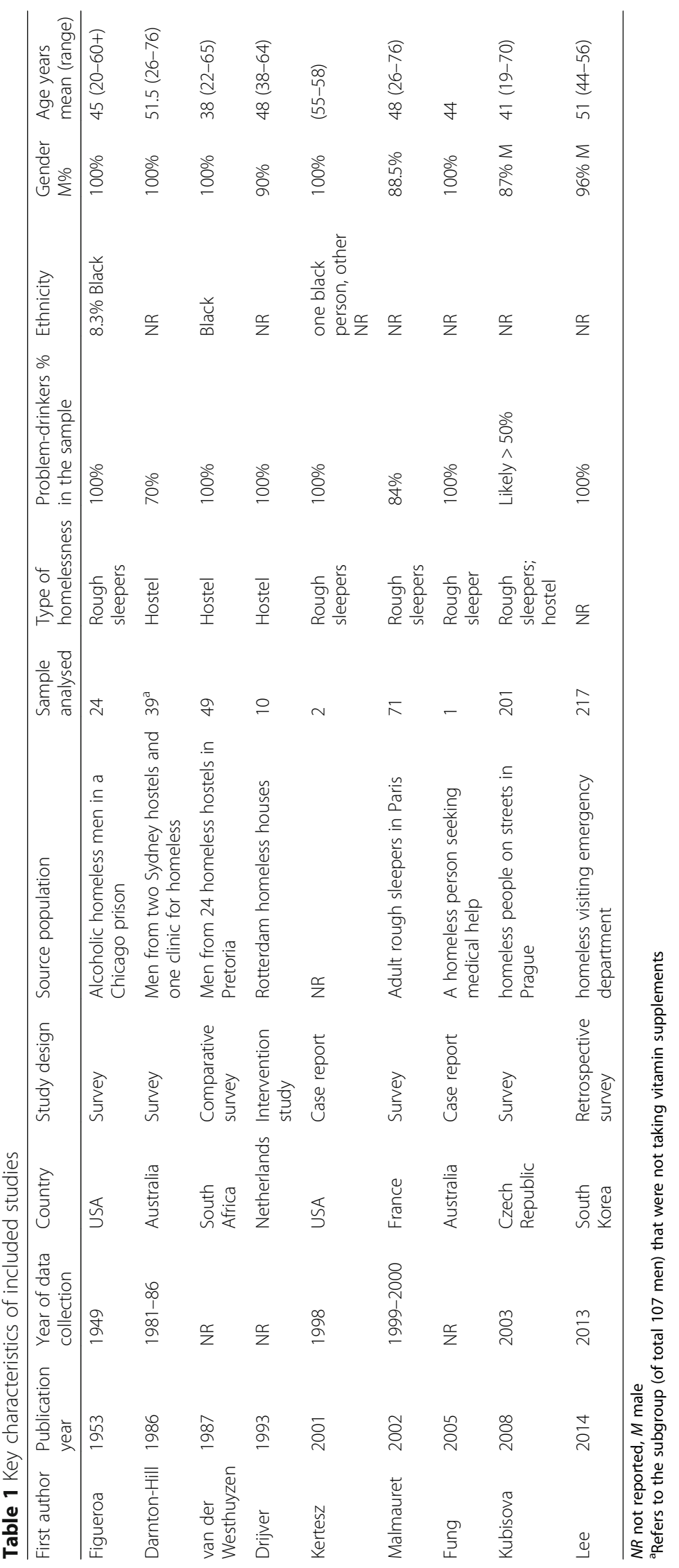




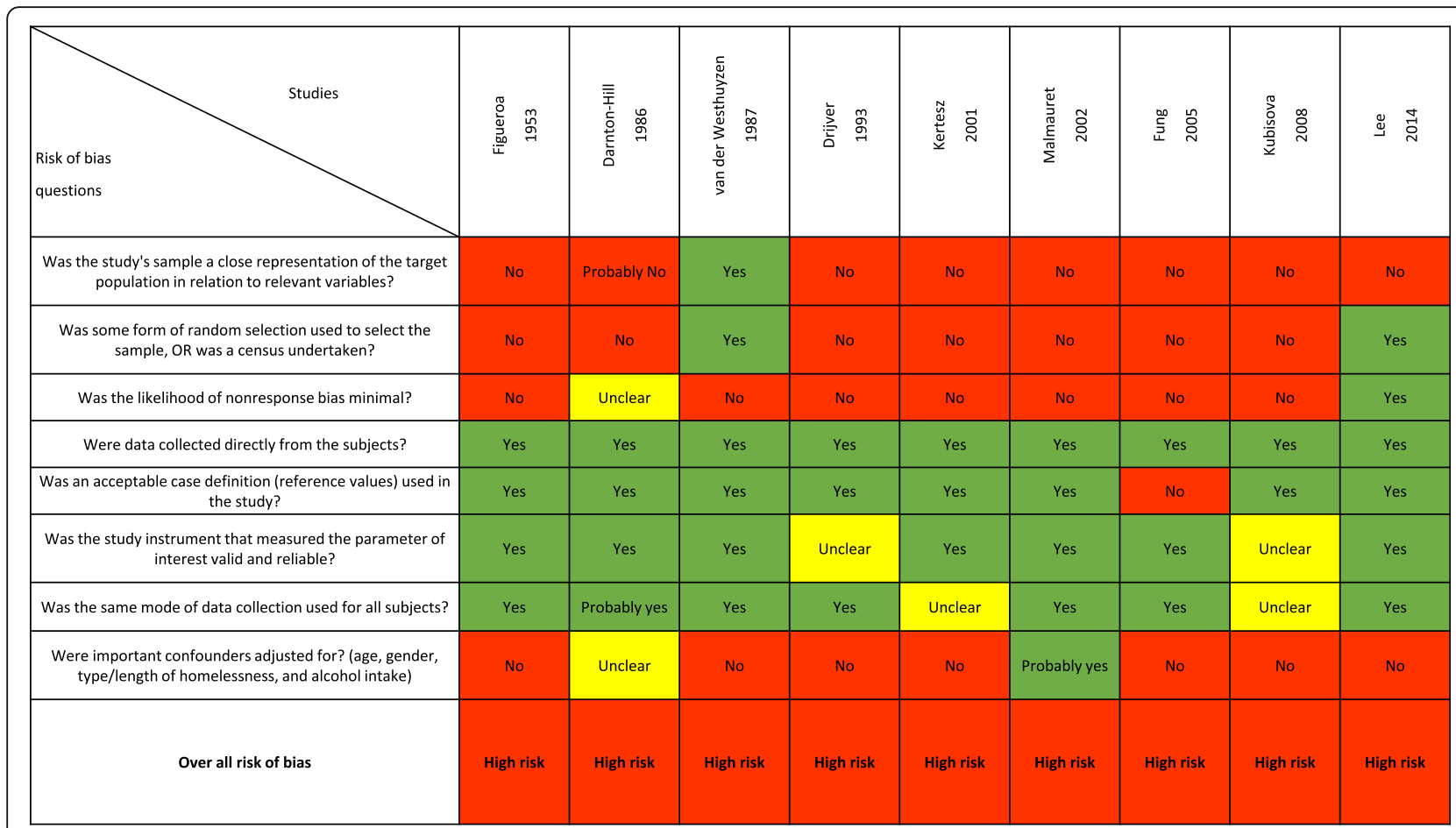

Fig. 3 Risk of bias across included studies

be deficient. However, the two more recent studies $[19,21]$ found just $2 \%$ and $6 \%$ were deficient, respectively. For vitamins B2, B6, B9, B12 and vitamin E the prevalence of deficiencies was low (0 to 23\%). In the single study assessing vitamin A $44 \%$ of the participants were found to be deficient [21].

Kertesz 2001 [24] reported two cases of clinically diagnosed pellagra but did not measure niacin (B3) levels. Both patients had avoided meals and shelter for several months and relied largely on alcohol for sustenance and both recovered with food and niacin supplements. The single case in Fung 2005 had also lived on several litres of wine a day for a year. Vitamin status was not checked in this person but the patient was found to be anaemic. The proportion with anaemia was reported in one other study [17]. Mean haemoglobin levels were reported in three other studies, all within normal range, with only one study reporting data separately for women and men [18]. Hypoalbuminemia (deficient serum albumin) was reported in three studies with the proportion ranging from 0 to $26 \%$.

We did not observe any obvious differences in nutritional deficiencies between different subgroups, e.g. in rough sleepers vs hostel dwellers. Similarly, since only single studies were identified from most locations, no clear difference was seen by country. We also presented graphically the prevalence of deficiencies for all vitamins, as reported in individual studies, to illustrate variation across populations and time (See Additional file 2).

\section{Discussion}

\section{Summary of findings}

We found that nutritional deficiencies have been observed to a variable extent in the homeless problemdrinking populations. Traditionally, vitamin B1 deficiency has been associated with this population and this is consistent with the findings of the two studies from the 1980s. However, in more recent years vitamin C deficiency appears to be more common [13, 19, 21]. The evidence however is of low quality, heterogeneous, and based on few studies, with only one [19] from this decade. This precludes effective use of these data to inform current clinical or public health practice. In addition, no studies measured vitamins B3, B5, B7, D, and K. Considering the evidence on global vitamin D deficiency [25] and its recommended supplementation [26] it is possible that the need for its supplementation also exists in homeless drinkers. The single study measuring vitamin $A$ and $E$ found a significant minority of the participants below reference levels [21].

\section{Comparison with previous work}

To our knowledge this is the first systematic review that evaluated deficiencies in this specific population. Our search was not limited by date or language allowing a comprehensive assessment of research across time and locations. Additionally, an extensive grey literature search ensured that we captured all relevant research pertaining to this population. 


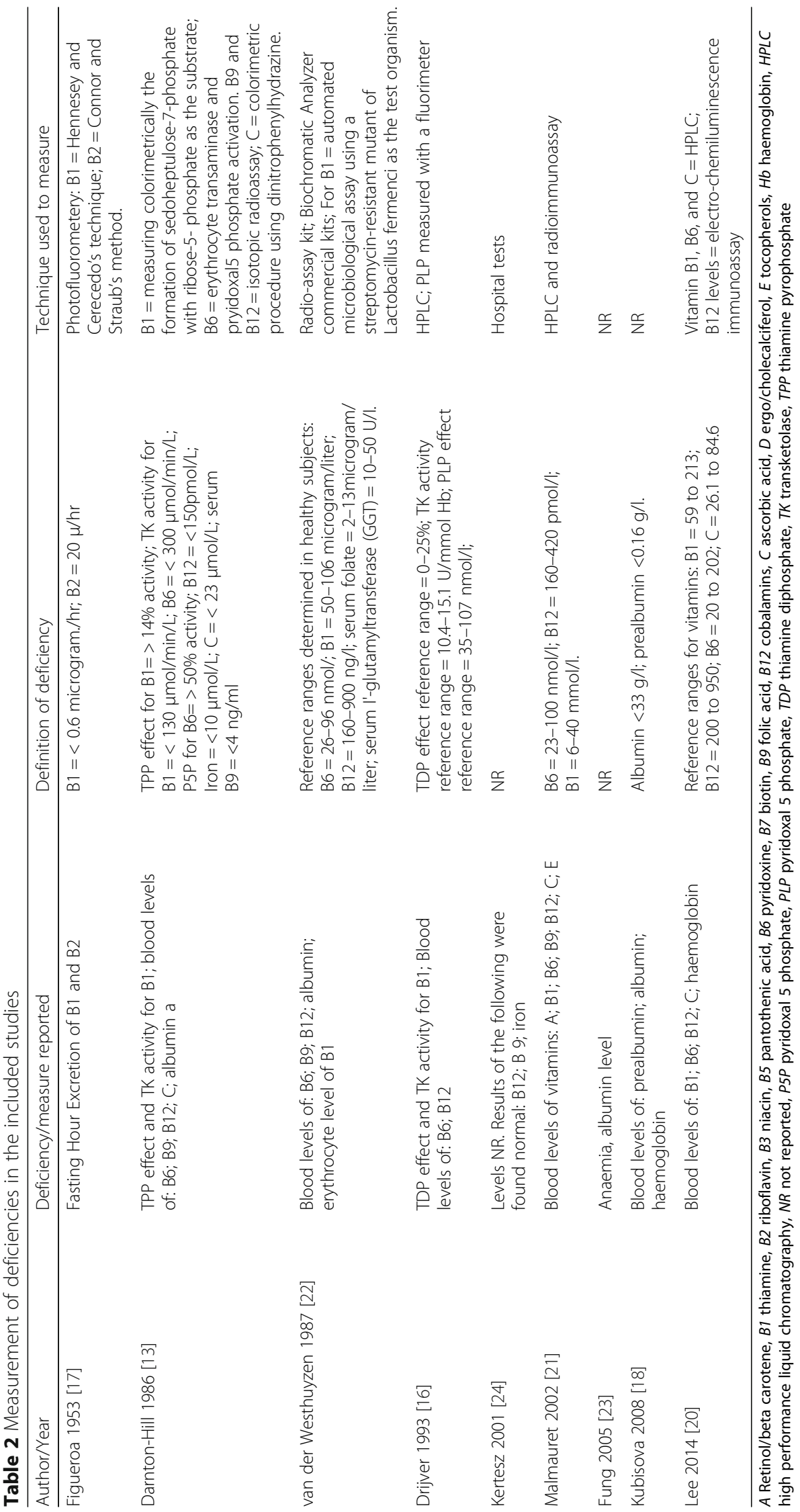




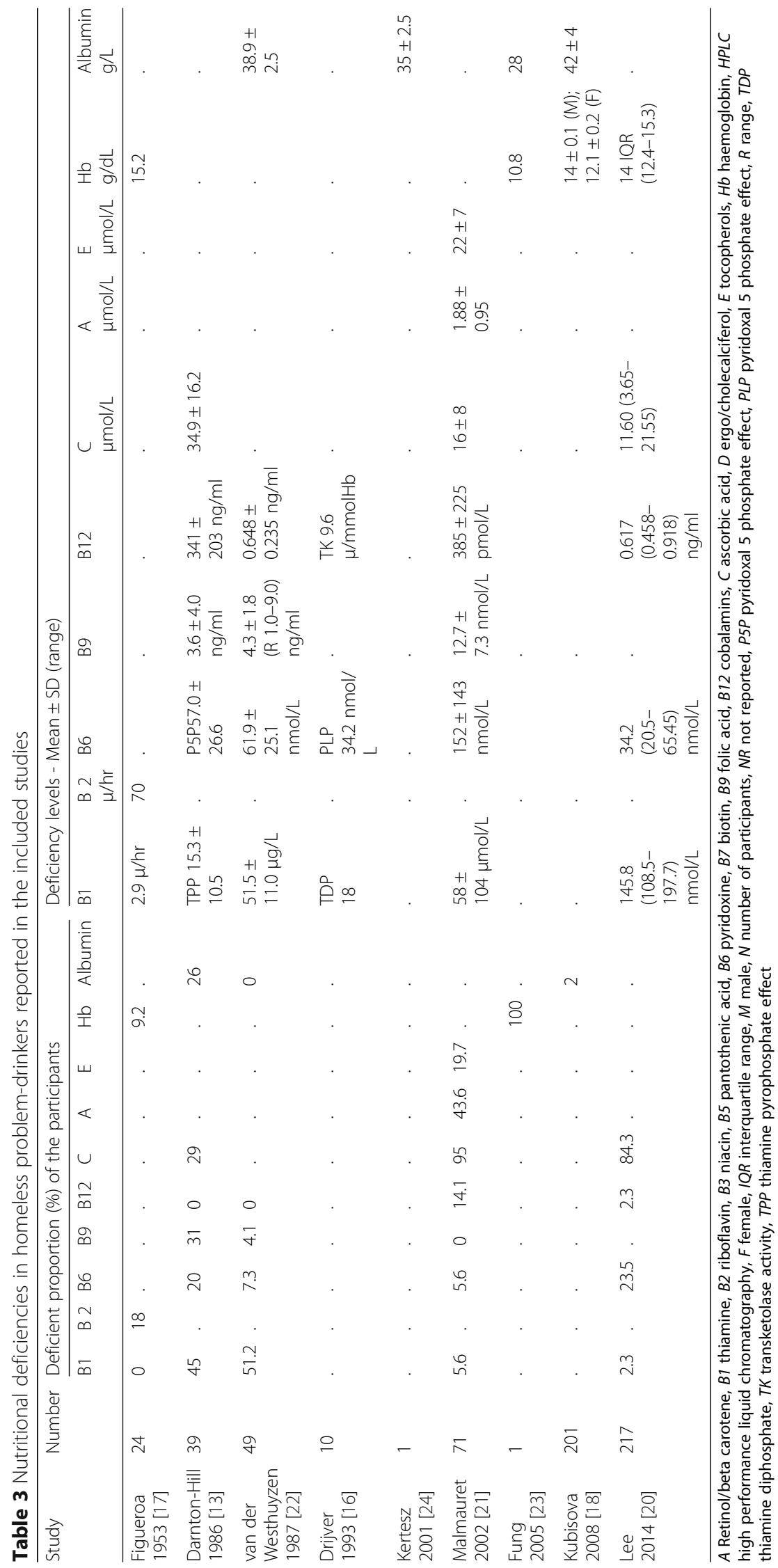


One previous systematic review [27] reported on the nutrition profile of the adult homeless in Paris. This review, based on a single study [21], found vitamin $\mathrm{C}$ to be an important deficiency for the homeless, in line with our findings. The majority of traditional reviews on alcohol-related nutrient deficiencies considered problem-drinking in the general population alone, and not in the homeless subgroup. All of these recommended further studies with better assessment of deficiencies [28-32]. Two reviews that have addressed the homeless subgroup of problem drinkers [2,33] also concluded that better assessment of their nutritional status was needed.

\section{Limitations}

The full range and severity of deficiencies may differ from those we found. This is partly because diets have changed over time in the general population [34, 35] and homeless heavy drinkers today are probably eating differently from those in the previous century. Also, the studies almost always included a convenient, rather than representative sample. Thus the proportion of homeless with a deficiency may be significantly higher (or lower) than that seen in the included studies.

The studies were few and varied. None of the included studies were at low risk of bias. The study with least risk of bias was the most recent study, from South Korea [19]. It is also important to consider applicability (external validity) of study findings to policy makers' circumstances. For example, given that dietary habits and levels of alcohol consumption in homeless populations in South Korea are likely to be different to those in Europe or North America, this study is unlikely to provide information relevant to European policy makers, and vice versa. There are variations in diets from one part of the world to another as well as changes in diets over time [36, 37]. Similar to diet variation across time and region, fortification practices have varied within and across regions in the past $[38,39]$. While most participants in individual studies were from hostels or shelters, these may not represent the majority who do not access these services [40] and there were few studies which included rough sleepers. All these factors resulted in relatively sparse and unreliable evidence being available for interpretation and our findings are limited by this.

Furthermore, the measurement of vitamin deficiencies has apparently also evolved over time and the reference ranges vary with methods and techniques used, and across populations [41-44]. It was therefore difficult to interpret (and inappropriate to pool) data across studies. This field may benefit from an international initiative to reach expert consensus on standardized definitions and thresholds for nutritional deficiencies. This could lead to future studies that are more informative to policy makers. Similar initiatives to develop core outcome sets for clinical trials have led to subsequent trials assessing comparable and relevant outcomes and thus contributing more meaningfully to the overall body of evidence in their fields $[45,46]$.

The complications of nutritional deficiencies in homeless problem-drinkers can be avoided if deficiencies are addressed early. A practical intervention would be a fortified food or supplement distributed to the homeless heavy drinkers. Such an intervention would work best when tailored to specific deficiencies in the target group. Provision of vitamin rich packets of chocolate paste has been found acceptable to rough sleepers in Paris [47] but there was no assessment of any resulting change in nutritional status. However, the included Darnton-Hill study [13] showed that providing supplements can change deficiency levels in this population.

Until a tailored nutritional or other relevant intervention can be developed to provide a long term solution, existing evidence can be used to improve the short term outcomes relevant to a healthcare system and society and to the homeless drinkers who suffer from deficiencies. Provision of vitamin supplements may prove useful [13, 48]. Fortification of beer [22, 49-51] although tempting, constitutes an ethical dilemma of turning a 'bad product' in to a 'super food' [52]. The idea is however not very different from needle exchange programs - the final aim being the prevention of disease when the risky behaviour cannot always be stopped [53, 54].

The available evidence is not sufficient to inform the development of a public health intervention for homeless drinkers. For example, the findings do not tell us which nutrients are lacking (and should be targeted) nor to what extent these deficiencies exist in the homeless heavy drinkers in specific countries today. A carefully designed study including a representative population in a specific country would be more useful to inform the development of such an intervention. The available data from the included studies did not allow us to draw conclusions on the importance of social factors (such as poverty, mental health, or family breakdown) but we acknowledge that addressing these issues could be part of the solution.

A representative sample is essential when studying prevalence. Homeless people are not easy to reach however, [55] so it may be practical to carry out a registrystudy of recent hospital/clinic records of the attending homeless problem-drinking patients. Such an analysis would be more representative than a small convenient sample. 


\section{Conclusions}

In summary, the limited, low quality, and relatively old evidence suggests that homeless problem-drinkers may be deficient in vitamin $\mathrm{C}$, thiamine, and other nutrients to varying levels. However, this is not a complete picture. Further well conducted studies are needed to inform public health interventions to improve deficiencies. But any future studies will be more useful if they are informed by expert consensus on definitions and thresholds for nutritional deficiencies, leading to comparable outcome measures across studies. Studies carried out in one country or community may not necessarily be suitable for informing public health policy in a different setting due to the differences in access to food, shelter and services, levels of alcohol consumption or local food fortification practices.

\section{Additional files}

Additional file 1: List of excluded studies. (PDF $338 \mathrm{~kb}$ )

Additional file 2: Graphic presentation of vitamin deficiencies in included studies. (JPG $579 \mathrm{~kb}$ )

\section{Abbreviations}

A\&E: Accident and emergency; B1: Thiamine; B12: Cobalamins; B2: Riboflavin; B3: Niacin; B5: Pantothenic acid; B6: Pyridoxine; B7: Biotin; B9: Folic acid; F: Female; Hb: Haemoglobin; HPLC: High performance liquid chromatography; IQR: Interquartile range; M: Male; NR: Not reported; P5P: Pyridoxal 5 phosphate; PLP: Pyridoxal 5 phosphate; TDP: Thiamine diphosphate; TK: Transketolase; TPP: Thiamine pyrophosphate; Vit A: Retinol/ beta carotene; Vit C: Ascorbic acid; Vit D: Ergo/cholecalciferol; Vit E: Tocopherols

\section{Acknowledgements}

Not applicable.

\section{Funding}

This work was supported by National Institute for Health Research (NIHR) Collaboration for Leadership in Applied Health Research and Care West (CLAHRC West) at University Hospitals Bristol NHS Foundation Trust. The views expressed are those of the authors and not necessarily those of the $\mathrm{NHS}$, the NIHR or the Department of Health.

\section{Availability of data and materials}

All data generated or analysed during this study are included in this published article [and its supplementary information files].

\section{Authors' contributions}

HT KP, CF, JS contributed to protocol development, AR developed and conducted searches. SI, JJ and JS conducted screening, selection, data extraction and risk of bias assessments. SI, JS, KP, CF and AB interpreted findings. All authors read and approved the final manuscript.

\section{Competing interests}

The authors declare that they have no competing interests.

\section{Consent for publication}

Not applicable.

Ethics approval and consent to participate Not applicable.

\section{Publisher's Note}

Springer Nature remains neutral with regard to jurisdictional claims in published maps and institutional affiliations.

\section{Author details}

${ }^{1}$ The National Institute for Health Research Collaboration for Leadership in Applied Health Research and Care West (NIHR CLAHRC West), University Hospitals Bristol NHS Foundation Trust, 9th Floor Whitefriars, Lewins Mead, Bristol BS1 2NT, UK. ${ }^{2}$ School of Social and Community Medicine, University of Bristol, Bristol, UK. ${ }^{3}$ Bristol City Council, St Annes House, Bristol BS4 4AB, UK. ${ }^{4}$ Compass Health, The Compass Centre, 1 Jamaica Street, Bristol BS2 8JP, UK.

${ }^{5}$ Research and Development Unit, The Salvation Army, London SE1 6BN, UK.

Received: 18 January 2017 Accepted: 24 April 2017

Published online: 05 May 2017

\section{References}

1. Homeless Link. The unhealthy state of homelessness. In: Health Audit Results 2014. London: Homeless Link; 2014. p. 23.

2. Salaspuro M. Nutrient intake and nutritional status in alcoholics. Alcohol Alcohol. 1993;28:85-8.

3. de la Monte SM, Kril JJ. Human alcohol-related neuropathology. Acta Neuropathol. 2014;127:71-90.

4. Thomson AD, Cook CCH, Touquet R, Henry JA. The Royal College of Physicians Report on Alcohol: Guidelines For Managing Wernicke's Encephalopathy In The Accident And Emergency Department. Alcohol Alcohol. 2002;37:513-21.

5. Edmunds A, Hill PS, McCormick B, Poteliakhoff B, Rose A, Scholes M, White J, Healthcare for Single Homeless People. London: The Department of health; 2010. https://www.housinglin.org.uk/_assets/Resources/Housing/Support_ materials/Other_reports_and_guidance/Healthcare_for_single_homeless_ people.pdf.

6. Thorley H, Porter K, Fleming C, Jones T, Kesten J, Marques E, Richards A, Savovic J. Interventions for preventing or treating malnutrition in problem drinkers who are homeless or vulnerably housed: protocol for a systematic review. Syst Rev. 2015;4:131.

7. Legal definition of homelessness. [http://england.shelter.org.uk/get_advice/ homelessness/homelessness_-_an_introduction/legal_definition_of_ homelessness]. Accessed 23 July 2015.

8. Hoy D, Brooks P, Woolf A, Blyth F, March L, Bain C, Baker P, Smith E, Buchbinder R. Assessing risk of bias in prevalence studies: modification of an existing tool and evidence of interrater agreement. J Clin Epidemiol. 2012;65:934-9.

9. Loney PL, Chambers LW, Bennett KJ, Roberts JG, Stratford PW. Critical appraisal of the health research literature: prevalence or incidence of a health problem. Chronic Dis Can. 1998;19:170-6.

10. Boyle MH. Guidelines for evaluating prevalence studies. Evid Based Ment Health. 1998;1:37-9.

11. Munn Z, Moola S, Riitano D, Lisy K. The development of a critical appraisal tool for use in systematic reviews addressing questions of prevalence. Int J Health Policy Manag. 2014;3:123-8.

12. Darnton-Hill I, Ash S. Dietary and alcohol intake patterns of a sample of homeless men in Sydney, Australia. J Hum Nutr Diet. 1988;1:397-408.

13. Darnton-Hill I, Sriskandarajah N, Craig GK, Truswell AS. Vitamin status of homeless men, Proceedings of the Nutrition Society of Australia, vol. 11. 1986.

14. Darnton-Hill I, Sriskandarajah N, Stewart PM, Craig G, Truswell AS. Vitamin supplementation and nutritional status in homeless men. Aust J Public Health. 1993;17:246-51.

15. Darnton-Hill I, Truswell AS. Thiamin status of a sample of homeless clinic attenders in Sydney. Med J Aust. 1990:152:5-9.

16. Drijver $E$, Van Strik R. Influence of intramuscular vitamin-B supplementation on TK activity and TDP-effect in erythrocytes in alcoholics. [Dutch]. TGO Tijdschrift voor Therapie Geneesmiddel en Onderzoek. 1993;18:132-7.

17. Figueroa WG, Sargent F, Imperiale L, Morey GR, Paynter CR, Vorhaus L, Kark RM. Lack of avitaminosis among alcoholics: its relation to fortification of cereal products and the general nutritional status of the population. J Clin Nutr. 1953:1:179-99.

18. Kubisova D, Dlouhy P, Rambouskova J, Andel M. Absence of protein-energy malnutrition in Prague homeless. Int J Public Health. 2008;53:57-63.

19. Lee HJ, Shin J, Hong K, Jung JH. Vitamin C Deficiency of Korean Homeless Patients Visiting to Emergency Department with Acute Alcohol Intoxication. J Korean Med Sci. 2015;30:1874-80. 
20. Lee HJ, Shin JH, Kang E, Jung J, Kim DK. Vitamin B and C levels of homeless patients who visit the emergency department with alcohol ingestion. Crit Care. 2014;18:S155.

21. Malmauret L, Leblanc J, Cuvelier I, Verger P. Dietary intakes and vitamin status of a sample of homeless people in Paris. Eur J Clin Nutr. 2002;56: 313-20.

22. van der Westhuyzen J, Davis RE, Icke GC, Metz J. Thiamine deficiency in black male hostel-dwellers. The need for thiamine supplementation of sorghum beer. S Afr Med J. 1987;71:231-4.

23. Fung AT, Rimmer J. Hypophosphataemia secondary to oral refeeding syndrome in a patient with long-term alcohol misuse. Med J Aust. 2005;183:324-6.

24. Kertesz SG. Pellagra in 2 homeless men. Mayo Clin Proc. 2001;76:315-8.

25. Mithal A, Wahl DA, Bonjour JP, Burckhardt P, Dawson-Hughes B, Eisman JA, Fuleihan GE, Josse RG, Lips P, Morales-Torres J, Advisors IOFCS. Global vitamin D status and determinants of hypovitaminosis D. Osteoporos Int. 2009;20:1807-20.

26. SACN. Vitamin D and Health. UK: Scientific Advisory Committee on Nutrition; 2016.

27. Kettaneh A, Stirnemann J, Fain O, Letellier E, Thomas M. [Micronutrients status of lle-de-France inhabitants. A literature review]. Rev Med Interne. 2004;25:507-13.

28. Leevy CM, Moroianu SA. Nutritional aspects of alcoholic liver disease. Clin Liver Dis. 2005;9:67-81.

29. Smith JW. Medical manifestations of alcoholism in the elderly. Int J Addict. 1995;30:1749-98

30. Auerhahn C. Recognition and management of alcohol-related nutritional deficiencies. Nurse Pract. 1992;17:40. 43-44, 49.

31. Klein S, Iber FL. Alcoholism and associated malnutrition in the elderly. Nutrition. 1991;7:75-9.

32. Lieber CS. The influence of alcohol on nutritional status. Nutr Rev. 1988;46: 241-54

33. Wiecha JL, Dwyer JT, Dunn-Strohecker M. Nutrition and health services needs among the homeless. Public Health Rep. 1991;106:364-74.

34. Foster R, Lunn J. 40th Anniversary Briefing Paper: Food availability and our changing diet. Nutr Bull. 2007:32:187-249.

35. Steyn NP, Bradshaw D, Norman R, Joubert JD, Schneider M, Steyn K. Dietary changes and the health transition in South Africa: implications for health policy. In: Division NaCP, editor. The double burden of malnutrition Case studies from six developing countries. Rome: Food and Agriculture Organization of the United Nations; 2006. p. 259-304.

36. Imamura F, Micha R, Khatibzadeh S, Fahimi S, Shi P, Powles J, Mozaffarian D, on behalf of the Global Burden of Diseases N, Chronic Diseases Expert G. Dietary quality among men and women in 187 countries in 1990 and 2010: a systematic assessment. Lancet Glob Health. 2015;3:e132-42.

37. Micha R, Khatibzadeh S, Shi P, Fahimi S, Lim S, Andrews KG, Engell RE, Powles J, Ezzati M, Mozaffarian D, et al. Global, regional, and national consumption levels of dietary fats and oils in 1990 and 2010: a systematic analysis including 266 country-specific nutrition surveys. BMJ. 2014;348: g2272.

38. Darnton-Hill I. Overview: Rationale and Elements of a Successful FoodFortification Programme. Food Nutr Bull. 1998;19:92-100.

39. Fletcher RJ, Bell IP, Lambert JP. Public health aspects of food fortification: a question of balance. Proc Nutr Soc. 2007;63:605-14.

40. Reeve K, Batty E. The hidden truth about homelessness. London: Crisis; 2011.

41. Reijnierse GLA, Van Der Horst AR, De Kloet K, Voorhorst CD. A radiochemical method for the determination of transketolase activity in erythrocyte hemolysates. Clin Chim Acta. 1978;90:259-68.

42. Ihara HHA, Wang L, Okada M, Hashizume N. Reference values for whole blood thiamine and thiamine phosphate esters in Japanese adults. J Anal Bio-Sci. 2005;28:241-6.

43. Itokawa $\mathrm{Y}$, Hashizume $\mathrm{N}$, Asano M, Igarashi O, Mino M, Ihara H, Ishiwata $\mathrm{Y}$, Kadowaki H, Kubota N, Okazaki M, et al. Proposed standard for human blood vitamin B1 value using HPLC. The Committee for Vitamin Laboratory Standards, Japan. Biofactors. 1999;10:295-9.

44. McLean $E$, de Benoist $B$, Allen LH. Review of the magnitude of folate and vitamin B-12 deficiencies worldwide. Food Nutr Bull. 2008;29:S38-51.

45. Williamson P, Altman D, Blazeby J, Clarke M, Gargon E. Driving up the Quality and Relevance of Research Through the Use of Agreed Core Outcomes. J Health Serv Res Policy. 2012;17:1-2.
46. Kirkham JJ, Boers M, Tugwell P, Clarke M, Williamson PR. Outcome measures in rheumatoid arthritis randomised trials over the last 50 years. Trials. 2013; 14:324.

47. Darmon N. A fortified street food to prevent nutritional deficiencies in homeless men in France. J Am Coll Nutr. 2009;28:196-202.

48. Manzardo AM. Clinical outcomes associated with benfotiamine treatment in chronic, severe alcohol dependence. Alcohol Clin Exp Res. 2014;38:310A.

49. Finlay-Jones R. Should thiamine be added to beer? Aust N Z J Psychiatry. 1986;20:3-6.

50. Centerwall BS, Criqui MH. Prevention of the Wernicke-Korsakoff syndrome: a cost-benefit analysis. N Engl J Med. 1978;299:285-9.

51. Fischer AJ, Yellowlees PM. Prevention of the Wernicke-Korsakoff syndrome in Australia: a cost-benefit analysis. Med J Aust. 1989;150:311-3. 317.

52. Kamien $\mathrm{M}$. The repeating history of objections to the fortification of bread and alcohol: from iron filings to folic acid. Med J Aust. 2006;184:638-40.

53. Wodak A, Cooney A. Do Needle Syringe Programs Reduce HIV Infection Among Injecting Drug Users: A Comprehensive Review of the International Evidence. Subst Use Misuse. 2006;41:777-813.

54. Kleinig J. Thinking Ethically About Needle and Syringe Programs. Subst Use Misuse. 2006;41:815-25.

55. Gelberg L, Linn LS. Social and physical health of homeless adults previously treated for mental health problems. Hosp Community Psychiatry. 1988;39: 510-6.

\section{Submit your next manuscript to BioMed Central and we will help you at every step:}

- We accept pre-submission inquiries

- Our selector tool helps you to find the most relevant journal

- We provide round the clock customer support

- Convenient online submission

- Thorough peer review

- Inclusion in PubMed and all major indexing services

- Maximum visibility for your research

Submit your manuscript at www.biomedcentral.com/submit
) Biomed Central 